\title{
Construction of 3D Arrays of Cylindrically Hierarchical Structures with ZnO Nanorods Hydrothermally Synthesized on Optical Fiber Cores
}

\author{
Weixuan Jing, Han Qi, Jiafan Shi, Zhuangde Jiang, Fan Zhou, and Yanyan Cheng \\ State Key Laboratory for Manufacturing Systems Engineering at Xian Jiaotong University, Xian 710049, China \\ Correspondence should be addressed to Weixuan Jing; wxjing@mail.xjtu.edu.cn
}

Received 10 September 2014; Accepted 19 November 2014; Published 9 December 2014

Academic Editor: Alireza Khataee

Copyright (C) 2014 Weixuan Jing et al. This is an open access article distributed under the Creative Commons Attribution License, which permits unrestricted use, distribution, and reproduction in any medium, provided the original work is properly cited.

With $\mathrm{ZnO}$ nanorods hydrothermally synthesized on manually assembled arrays of optical fiber cores, 3D arrays of $\mathrm{ZnO}$ nanorodbased cylindrically hierarchical structures with nominal pitch $250 \mu \mathrm{m}$ or $375 \mu \mathrm{m}$ were constructed. Based on micrographs of scanning electron microscopy and image processing operators of MATLAB software, the 3D arrays of cylindrically hierarchical structures were quantitatively characterized. The values of the actual diameters, the actual pitches, and the parallelism errors suggest that the process capability of the manual assembling is sufficient and the quality of the 3D arrays of cylindrically hierarchical structures is acceptable. The values of the characteristic parameters such as roughness, skewness, kurtosis, correlation length, and power spectrum density show that the surface morphologies of the cylindrically hierarchical structures not only were affected significantly by $\mathrm{Zn}^{2+}$ concentration of the growth solution but also were anisotropic due to different curvature radii of the optical fiber core at side and front view.

\section{Introduction}

$\mathrm{ZnO}$ nanorod has larger surface-to-volume ratio, wider band-gap, better electron transfer ability, higher isoelectrical point, and nontoxicity [1-4] and has been frequently employed to construct arrays of $\mathrm{ZnO}$ nanorod-based cylindrically hierarchical structures with larger specific surface area, expectant surface morphology, desirable wettability, and photocatalytic activity $[5,6]$. As an enzymatic electrode or a photocatalytic reactor, the array of cylindrically hierarchical structures was widely used to detect the concentration of glucose, cholesterol, urea, uric acid, hydroperoxide, and phenol [7] as well as to photodegrade methylene blue and methylene orange in waste water [8]. Synthesizing parameters of $\mathrm{ZnO}$ nanorods determine the surface morphology and the wettability of $\mathrm{ZnO}$ nanorod-based cylindrically hierarchical structure and eventually affect the enzyme immobilization of the aforementioned biosensors $[9,10]$ as well as the photodegradation efficiency of the target agents [11, 12]. Therefore, construction and quantitative characterization of various $3 \mathrm{D}$ arrays of $\mathrm{ZnO}$ nanorod-based cylindrically hierarchical structures with desirable surface morphology and larger surface area are prerequisite to the above mentioned biosensors and waste water treatment domains.

Construction of an array of $\mathrm{ZnO}$ nanorod-based cylindrically hierarchical structures includes the production of a cylinder array as the microscale smooth substrate and the synthesis of $\mathrm{ZnO}$ nanorods as the nanoscale rough surface. A stainless steel wire mesh with specific pitch and diameter has been frequently used as an array of microscale cylinders [13]. Of all approaches including hydrothermal synthesis [14], chemical vapor deposition (CVD) [15], and thermal evaporation method [16] to synthesize $\mathrm{ZnO}$ nanorods on a cylindrical substrate, simple hydrothermal synthesis not only is of low cost but also leads to $\mathrm{ZnO}$ nanorods of high quality. For instance, Li et al. [5] synthesized $\mathrm{ZnO}$ nanorods with CVD and hydrothermal routes, respectively, on stainless steel wire meshes not only to study the wettability switching under the alternation of UV irradiation and dark storage but also to separate water from oil. Vu et al. [17] hydrothermally synthesized $\mathrm{ZnO}$ nanostructures on a stainless steel wire mesh and investigated the effect of different synthesis parameters on the yield and the geometric dimensions of the samples. Besides 
the stainless steel mesh, a Cu wire mesh is also used as an array of microscale cylinders. For example, Lin et al. [18] attached $\mathrm{CdSe}$ nanoparticles to the surface of the radially orientated $\mathrm{ZnO}$ nanorods which were hydrothermally synthesized on a $\mathrm{Cu}$ wire mesh substrate as a wire-shaped electrode to improve the electrolyte mass transport and light harvesting. Nevertheless, the disadvantages of stainless steel and $\mathrm{Cu}$ meshes are that they consist of only two layers of microscale cylinders and have finite effective surface areas. As an ideal microscale cylinder and being of low cost, optical fiber cores have potential to be used to construct an array of more than 2 layers of microscale cylindrical substrates [19]. To our knowledge, synthesizing $\mathrm{ZnO}$ nanorods on an array of optical fiber cores with specific pitch has not been reported in other literatures.

In situ measurement route comparing the features of a scanning electron microscopy (SEM) micrograph with related scale bar is widely employed to coarsely estimate the limited geometric parameters of a $\mathrm{ZnO}$ nanorod-based cylindrically hierarchical structure. For instance, $\mathrm{Vu}$ et al. [8] measured the thicknesses of the $\mathrm{ZnO}$ nanorod film on a stainless steel mesh. Usman Ali et al. [20] approximately estimated the diameters and the lengths of $\mathrm{ZnO}$ nanorods synthesized on an Ag wire as a working electrode. Zhang and Jiao [21] roughly figured out the diameters of $\mathrm{ZnO}$ nanowires synthesized on carbon fibers. Similarly, Ehlert and Sodano [22] roughly estimated the diameters and the lengths of $\mathrm{ZnO}$ nanorods grown on polymer fibers, and Huang et al. [23] approximately derived the diameters and the lengths of $\mathrm{ZnO}$ nanorods synthesized on carboxylic acid functionalized aramid fibers. Since the line edges of the features are not extracted from the above mentioned SEM micrographs, the aforementioned measurement data are insufficient and the characterization parameters are deficient. This led to incomplete geometric characterization of these $\mathrm{ZnO}$ nanorod-based cylindrically hierarchical structures. Moreover with the in situ measurement route mentioned above, it is difficult to quantitatively characterize the surface morphology of any cylindrically hierarchical structure.

In this paper, 3D arrays of 2 and 3 layers of $\mathrm{ZnO}$ nanorodbased cylindrically hierarchical structures were constructed. More geometric parameters were introduced and the surface morphologies were quantitatively characterized. The process capability of the manual assembling was evaluated, and the influencing factors of the surface morphologies of the cylindrically hierarchical structures were identified.

\section{Experimental}

2.1. Materials and Apparatus. Absolute ethyl alcohol, anhydrous zinc acetate (Kemiou Chemical Reagent, China), sodium hydrate (Hedong Red Cliff Chemical Reagent Factory, China), zinc nitrate, hexamethylenetetramine, six hydrated zinc nitrate (Tianjin Fuchen Chemical Reagents Factory, China), and acetone are all analytical reagents. DI water $(18.25 \mathrm{M} \Omega \cdot \mathrm{cm})$, glass substrate (Sail Brand, Shenzhen Xinghonglian Sci. \& Tech. Co. Ltd., China), optical fiber

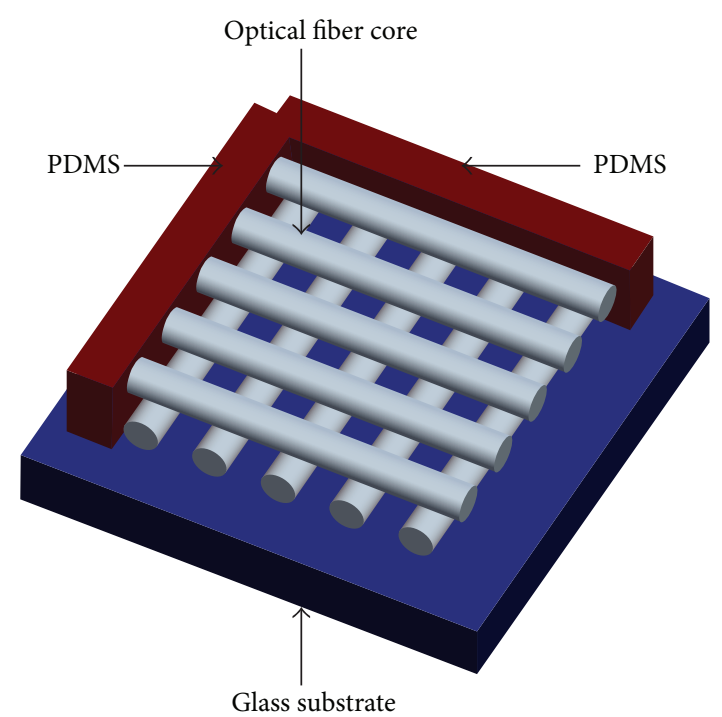

FIGURE 1: Schematic of a 3D array of 2 layers of optical fiber cores with nominal pitch $250 \mu \mathrm{m}$.

(Ф125 $\mu \mathrm{m}$ Quanzhou Anpon Company, China), and polydimethylsiloxane (PDMS) solvent and hardener (Sylgard184, Dow Corning Company, USA) were employed.

Water-bath (DK-98-IIA, Tasite, China), scanning electron microscopy (SU8010, Hitachi, Japan), drying oven (DZF-6020, Shanghai Yiheng Technical Co. Ltd., China), magnetic stirrer (BII-3, Shanghai Sile Automation Science \& Technology Co. Ltd., China), ultrasonic washer (KQ-100DE, Kunshan Ultrasonic Instruments Co. Ltd., China), superior spectral ultrapure water manufacturing system (UPR-II5/10T, Chengdu Ultrapure Technology Co. Ltd., China), and electronic balance (FA1004N, Changzhou Xingyun Electronic Equipment Co. Ltd., China) were used.

\subsection{Construction of the 3D Arrays of Cylindrically Hierarchical Structures}

2.2.1. Manually Assembling the 3D Arrays of Optical Fiber Cores. Dual-mode optical fibers with core diameter $\Phi 125 \mu \mathrm{m}$ and length $30 \mathrm{~mm}$ were immersed in acetone solution for $15 \mathrm{~min}$. The optical fiber cores were pulled out from the plastic sheaths, subjected to ultrasonic cleaning in absolute ethyl alcohol and DI water for $5 \mathrm{~min}$, respectively, and dried in air. These optical fiber cores were manually placed next to each other on a glass substrate and fixed securely at one end by PDMS with the ratio of solvent to hardener $10: 1$. The assembly of the optical fiber cores and the glass substrate were dried in the drying oven at $80^{\circ} \mathrm{C}$ for $30 \mathrm{~min}$. Every other one optical fiber core regarded as a positioning fixture was removed from the glass substrate; thus the 1st layer of optical fiber cores with nominal pitch $250 \mu \mathrm{m}$ was formed. Similarly, the 2 nd layer of optical fiber cores was vertically placed upon the 1st layer, and a 3D array of 2 layers of optical fiber cores with nominal pitch $250 \mu \mathrm{m}$ was produced; see Figure 1 . Once more, the 3 rd layer of optical fiber cores was vertically placed 
TABLE 1: Quantitative characterization of the 3D arrays of cylindrically hierarchical structures.

\begin{tabular}{lccccc}
\hline & \multicolumn{3}{c}{ The 3D arrays of cylindrically hierarchical structures } \\
& \multicolumn{2}{c}{ 2 layers with nominal pitch $250 \mu \mathrm{m}$} & \multicolumn{3}{c}{ 3 layers with nominal pitch 375 $\mu \mathrm{m}$} \\
& 1st layer & 2nd layer & 1st layer & 2nd layer & 3rd layer \\
\hline Actual diameter $(\mu \mathrm{m})$ & $\Phi 124.8 \pm 0.44$ & $\Phi 125.48 \pm 0.69$ & $\Phi 124.02 \pm 0.83$ & $\Phi 125.03 \pm 0.44$ & $\Phi 125.57 \pm 0.79$ \\
Actual pitch $(\mu \mathrm{m})$ & $248.44 \pm 8.16$ & $248.06 \pm 3.41$ & $385.59 \pm 11.98$ & 421.12 & $391.17 \pm 11.12$ \\
Parallelism error $(\mu \mathrm{m})$ & $0.45 \pm 0.59$ & $1.11 \pm 0.66$ & $1.00 \pm 0.822$ & $8.40 \pm 10.54$ & $5.32 \pm 4.69$ \\
\hline
\end{tabular}

upon the 2nd layer, and a 3D array of 3 layers of optical fiber cores with nominal pitch $250 \mu \mathrm{m}$ was generated.

The aforementioned construction process was repeated and every other two neighboring optical fiber cores regarded as positioning fixtures were removed; likewise, the $3 \mathrm{D}$ arrays of 2 and 3 layers of optical fiber cores with nominal pitch $375 \mu \mathrm{m}$ were formed, respectively.

\subsubsection{Synthesizing $\mathrm{ZnO}$ Nanorods on the $3 \mathrm{D}$ Arrays} of Optical Fiber Cores. Solid zinc acetate dihydrate $\left(\mathrm{Zn}\left(\mathrm{CH}_{3} \mathrm{COO}\right)_{2} \cdot 2 \mathrm{H}_{2} \mathrm{O}\right)$, sodium hydrate $(\mathrm{NaOH})$, and absolute ethyl alcohol were employed to prepare the $\mathrm{ZnO}$ seed solution of $\mathrm{Zn}^{2+}$ concentration $1 \mathrm{mM}$. Zinc nitrate $\left(\mathrm{Zn}\left(\mathrm{NO}_{3}\right)_{2} \cdot 6 \mathrm{H}_{2} \mathrm{O}\right)$, hexamethylenetetramine $\left(\mathrm{C}_{6} \mathrm{H}_{12} \mathrm{~N}_{4}\right)$, and DI water were used to produce the growth solution of $\mathrm{Zn}^{2+}$ concentrations 25, 40, 55, 70, 85, and $100 \mathrm{mM}$, respectively.

The $\mathrm{ZnO}$ seed solution of $2 \mathrm{~mL}$ was dropped on 6 optical fiber cores, and the optical fiber cores were annealed at $120^{\circ} \mathrm{C}$ for $10 \mathrm{~min}$ in the drying oven. These dropping and annealing processes were repeated twice more. The optical fiber cores were put into 6 beakers, respectively, and the growth solutions of $\mathrm{Zn}^{2+}$ concentrations 25, 40, 55, 70, 85, and $100 \mathrm{mM}$ were poured into these beakers quickly. The beakers were sealed with preservative films and then put into the water bath at $90^{\circ} \mathrm{C}$ for $2.5 \mathrm{~h}$. The optical fiber cores were taken out, cleaned with DI water for $5 \mathrm{~min}$ in the ultrasonic washer, and dried in air. Therefore, 6 cylindrically hierarchical structures with different surface morphologies were produced.

Similarly, the $\mathrm{ZnO}$ seed solution was deposited on the aforementioned 3D arrays of 2 and 3 layers of optical fiber cores with nominal pitches $250 \mu \mathrm{m}$ and $375 \mu \mathrm{m}$. The arrays were put into the growth solution of $\mathrm{Zn}^{2+}$ concentration $100 \mathrm{mM}$ at $90^{\circ} \mathrm{C}$ for $2.5 \mathrm{~h}$. Hence, the $3 \mathrm{D}$ arrays of 2 and 3 layers of cylindrically hierarchical structures with nominal pitches $250 \mu \mathrm{m}$ and $375 \mu \mathrm{m}$ were formed.

2.3. Quantitatively Characterizing the 3D Arrays of Cylindrically Hierarchical Structures. Based on SEM micrographs and filtration, line edge detection, and fitting operators of MATLAB software, not only the profiles of the 3D arrays of cylindrically hierarchical structures but also those of the cylindrically hierarchical structures at side and front view were extracted. In terms of these extracted profiles, the actual diameters, the actual pitches, and the parallelism errors of the $3 \mathrm{D}$ arrays of cylindrically hierarchical structures as well as the characteristic parameters of the surface morphologies of the cylindrically hierarchical structures were determined. The characteristic parameters include surface roughness $(\mathrm{Ra})$, skewness (Sk), kurtosis $(\mathrm{Ku})$, correlation length $(\zeta)$, and power spectrum density (PSD).

\section{Results and Discussion}

3.1. Characterization of the 3D Arrays of Cylindrically Hierarchical Structures. Figure 2 demonstrates the 3D arrays of 2 and 3 layers of cylindrically hierarchical structures with nominal pitches $250 \mu \mathrm{m}$ and $375 \mu \mathrm{m}$. Curves in red (the 1st layer) and in green (the 2nd layer) in Figure 2(a) are the extracted profiles of the 3D array of 2 layers of cylindrically hierarchical structures with nominal pitch $250 \mu \mathrm{m}$, whilst curves in red (the 1st layer), in green (the 2nd layer), and in yellow (the 3rd layer) in Figure 2(d) are those of the 3D array of 3 layers of cylindrically hierarchical structures with nominal pitch $375 \mu \mathrm{m}$. Circled letters A and B in Figure 2(a) and C, D, and E in Figure 2(d) are datum lines to evaluate the parallelism errors of corresponding line edges.

In light of these profiles and the datum lines, the actual diameters, the actual pitches, and the parallelism errors of the 3D arrays of 2 and 3 layers of cylindrically hierarchical structures were determined; see Table 1.

The actual diameters of cylindrically hierarchical structures in Figures 2(a) and 2(d) increased gradually from the bottom to the top layer. It is attributed to the square blindholes formed by the 3D array of the optical fiber cores along with the glass substrates; see regions in grey in Figure 3. These square blind-holes resulted in different $\mathrm{Zn}^{2+}$ diffusion rates of the growth solution to the surfaces of the optical fiber cores among the 1st, the $2 \mathrm{nd}$, and the 3rd layers. That is, $\mathrm{Zn}^{2+}$ concentration of the growth solution around the surfaces of optical fiber cores at the 1st layer was lower, that at the 2nd layer was low, and that at the 3rd layer was high. Hence, the growth rates of $\mathrm{ZnO}$ nanorods on the optical fiber cores increased from the 1st through the 2 nd and to the 3rd layer and resulted in increasing actual diameters of the cylindrically hierarchical structures from the bottom to the top layer.

The averages of the actual pitches of the 3D array of the cylindrically hierarchical structures in Figure 2(a) deviated less from nominal pitch $250 \mu \mathrm{m}$ than that in Figure 2(d) from nominal pitch $375 \mu \mathrm{m}$. The positioning fixture removing every other one optical fiber core resulted in less deformation of PDMS than taking away every other two neighboring optical fiber cores. Meanwhile, the more the deformation of PDMS is, the larger the actual pitch deviation is. This suggests that the process capability of manually assembling 


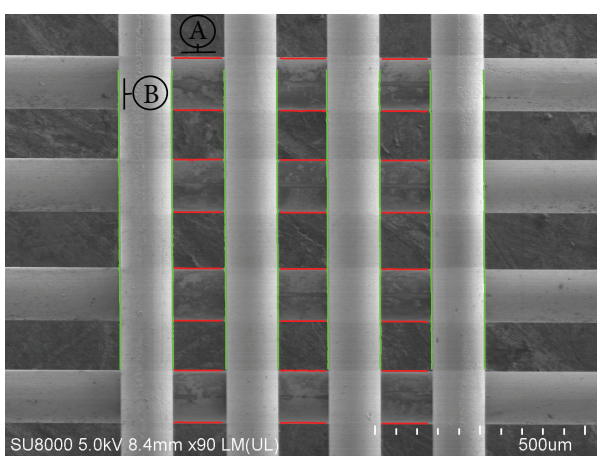

(a)

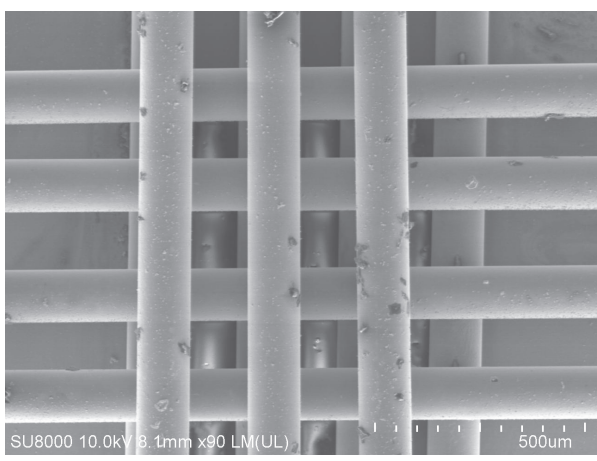

(c)

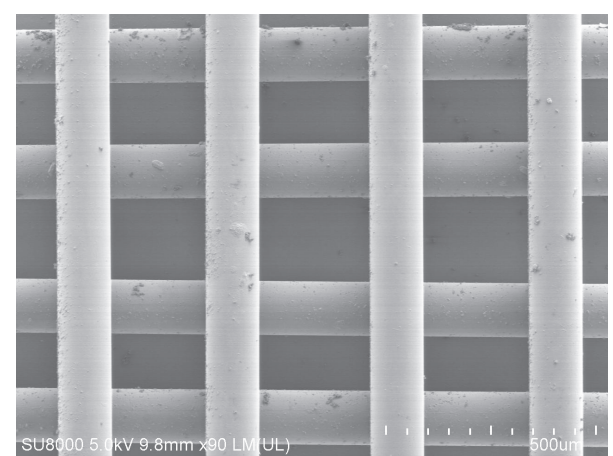

(b)

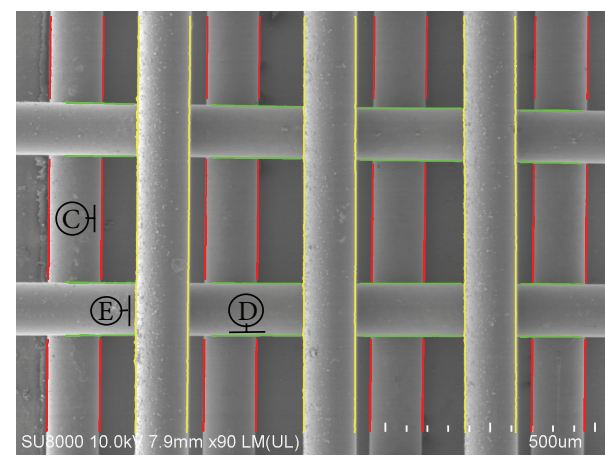

(d)

FIgure 2: The 3D arrays of cylindrically hierarchical structures with different layers and nominal pitches. (a) Two layers, nominal pitch $250 \mu \mathrm{m}$. (b) Two layers, nominal pitch $375 \mu \mathrm{m}$. (c) Three layers, nominal pitch $250 \mu \mathrm{m}$. (d) Three layers, nominal pitch $375 \mu \mathrm{m}$.

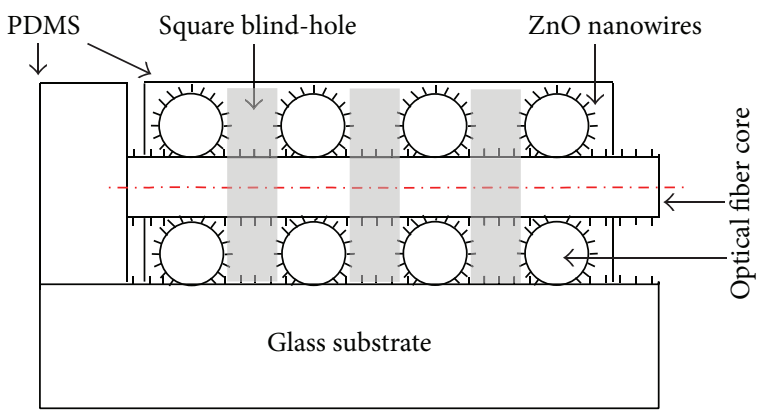

FIGURE 3: Schematic of the square blind-holes formed by the glass substrate and the optical fiber cores.

the 3D array of optical fiber cores with nominal pitch $250 \mu \mathrm{m}$ is better than that with nominal pitch $375 \mu \mathrm{m}$. Similar conclusion can be drawn from the parallelism errors of the cylindrically hierarchical structures relative to data A and B in Figure 2(a) as well as that with regard to data C, D, and E in Figure 2(d).

\subsection{Qualitative Characterization of the Surface Morphologies} of the Cylindrically Hierarchical Structures. For the cylindrically hierarchical structure produced at low $\mathrm{Zn}^{2+}$ concentration of the growth solution, hexagonal $\mathrm{ZnO}$ nanorods are evident and are randomly aligned, and the diameters are small (Figure 4(a)). As $\mathrm{Zn}^{2+}$ concentration of the growth solution gradually increased, the diameters of $\mathrm{ZnO}$ nanorods became larger, and the direction became less random (Figures 4(b) and 4(c)), even recrystallization among $\mathrm{ZnO}$ nanorods occurred (Figures 4(d) and 4(e)), and eventually $\mathrm{ZnO}$ nanostructures instead of $\mathrm{ZnO}$ nanorods appeared (Figure 4(f)).

The top left and the bottom left insets in Figure 4 demonstrate the cylindrically hierarchical structures at side and front view, respectively. Obviously $\mathrm{ZnO}$ nanorods were uniformly synthesized all around the smooth optical fiber cores and radially orientated in normal direction. This suggests that desirable rough surfaces of high spatial frequencies were superimposed on the smooth cylindrical surfaces of low spatial frequency and the cylindrically hierarchical structures with expectant surface morphologies were produced.

Figure 5 shows the XRD spectrum of $\mathrm{ZnO}$ nanorods in Figure $4(\mathrm{a})$. All ten peaks are indexed in $2 \theta$ range of $20^{\circ}$ to $80^{\circ}$ (JCPDS Card number 36-1451). This indicates that the orientation of $\mathrm{ZnO}$ nanorods is random to some extent. The strength of (002) peak is stronger than that of other peaks. This suggests that $\mathrm{ZnO}$ nanorods grew along $c$-axis and the crystallinity is high. The XRD analysis is in agreement with the SEM micrograph of $\mathrm{ZnO}$ nanorods in Figure 4(a). Similar conclusion between SEM micrographs of $\mathrm{ZnO}$ nanorods in Figures $4(\mathrm{~b})-4(\mathrm{f})$ and related XRD analysis can also be drawn.

Curves in red and in blue in both insets of Figures 4(a) and 4(f) are the extracted profiles of corresponding cylindrically hierarchical structures at side and front view. 


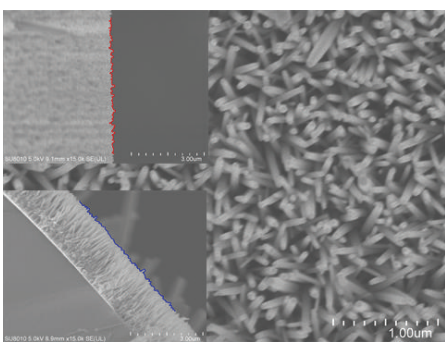

(a)

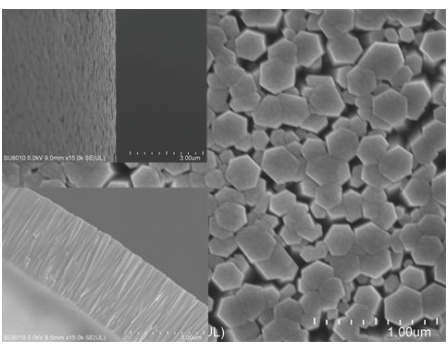

(d)

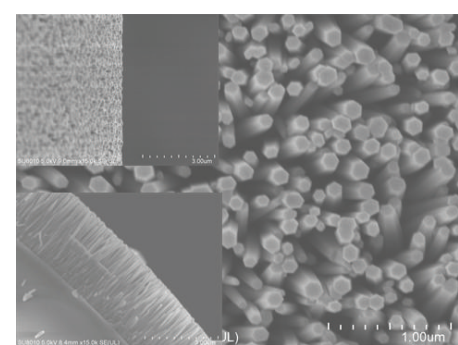

(b)

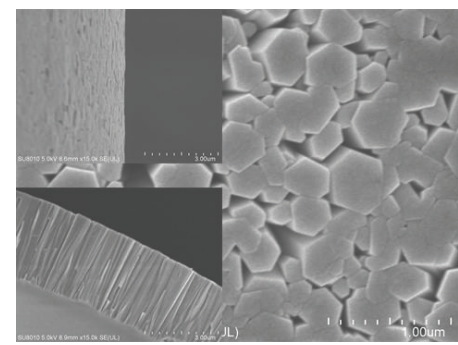

(e)

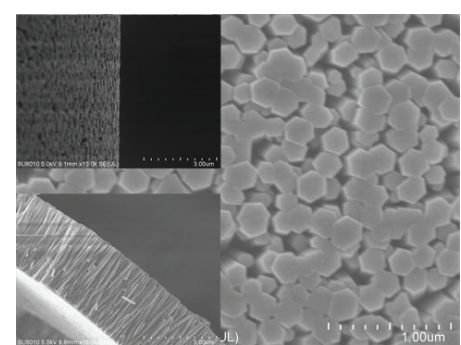

(c)

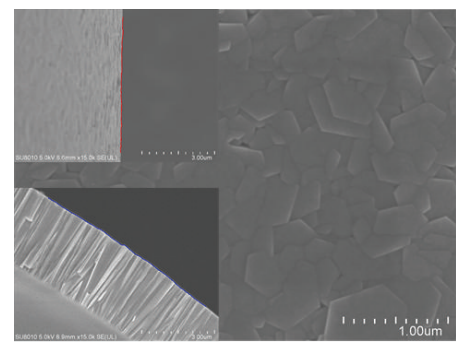

(f)

Figure 4: $\mathrm{ZnO}$ nanorod-based cylindrically hierarchical structures produced at $\mathrm{Zn}^{2+}$ concentrations $25 \mathrm{mM}$ (a), $40 \mathrm{mM}$ (b), $55 \mathrm{mM}$ (c), $70 \mathrm{mM}(\mathrm{d}), 85 \mathrm{mM}$ (d), and $100 \mathrm{mM}$ (f) of the growth solution, respectively.

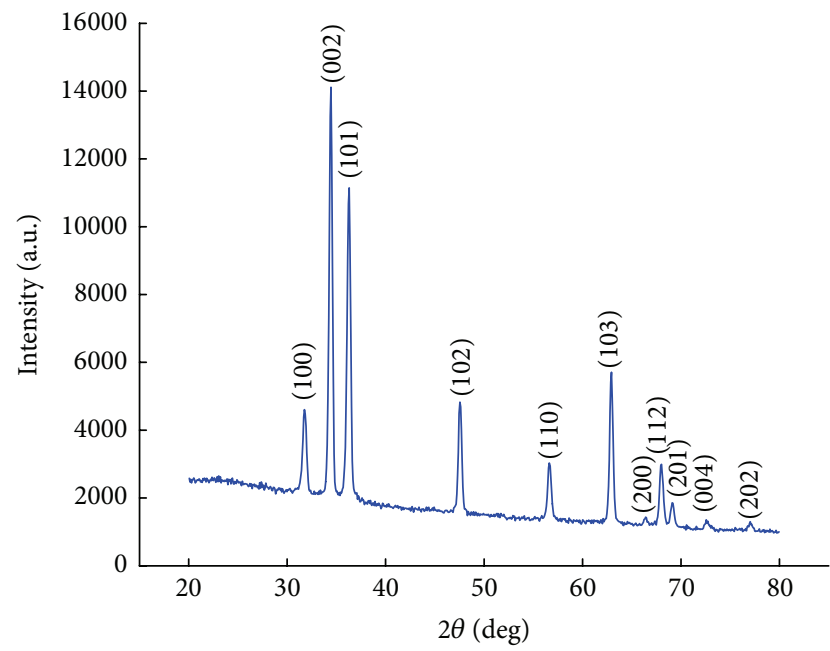

FIGURE 5: XRD profile of $\mathrm{ZnO}$ nanorods in Figure 4(a).

From the profiles at front view, the median curves were fitted. With the median curves subtracted from the profiles at front view, the surface morphologies of the cylindrically hierarchical structures at front view were obtained; see curves in blue in Figures 6(a) and 6(b). Curves in red in Figures 6(a) and 6(b) are the extracted profiles in red in top left insets of Figures 4(a) and 4(f) which represent the surface morphologies of the cylindrically hierarchical structures at side view.

Comparing the profiles in Figure 6(a) with corresponding ones in Figure 6(b), obviously, the fluctuation ranges of the surface heights of the former profiles are larger than those of the latter ones. This concludes that the larger the
$\mathrm{Zn}^{2+}$ concentration of the growth solution, the smoother the profiles at side and front view. For both hierarchical structures, the profiles in blue almost envelop that in red. This suggests that the profiles at front view are rougher than those at side view. That is, anisotropic surface morphologies of the cylindrically hierarchical structures appeared.

3.3. Quantitative Characterization of the Surface Morphologies of the Cylindrically Hierarchical Structures. Similarly, the profiles of the surface morphologies of the cylindrically hierarchical structures in Figures 4(b) 4(e) were extracted too. According to these profiles, the characteristic parameters Ra, $\zeta$, Sk, Ku, and PSD of corresponding surface morphologies were determined.

3.3.1. Characteristic Parameters $R a, \zeta, S k$, and $K u$. Figure 7(a) exhibits that the increase of $\mathrm{Zn}^{2+}$ concentration of the growth solution resulted in the decrease of both $\mathrm{Ra}_{\text {front }}$ and $\mathrm{Ra}_{\text {side }}$ values. It suggests that the profiles of the cylindrically hierarchical structures at front and side view gradually became smoother. With $\mathrm{Zn}^{2+}$ concentration of the growth solution increasing, the diameters of $\mathrm{ZnO}$ nanorods gradually became larger. This led to the decreasing sizes of the pits among $\mathrm{ZnO}$ nanorods, gave rise to better aligned $\mathrm{ZnO}$ nanorods or even $\mathrm{ZnO}$ nanostructures, and produced smoother surfaces. Moreover, $\mathrm{Ra}_{\text {front }}$ values are slightly larger than corresponding $\mathrm{Ra}_{\text {side }}$. This suggests that the profiles at front view are a bit rougher than the ones at side view and anisotropic surface morphologies were formed. This is attributed to the difference of the curvature radii of the optical fiber core at front and side view. The curvature radius of the optical fiber core at front view is significantly less than that at side view. This resulted in radially orientated $\mathrm{ZnO}$ nanorods from the surfaces of the 


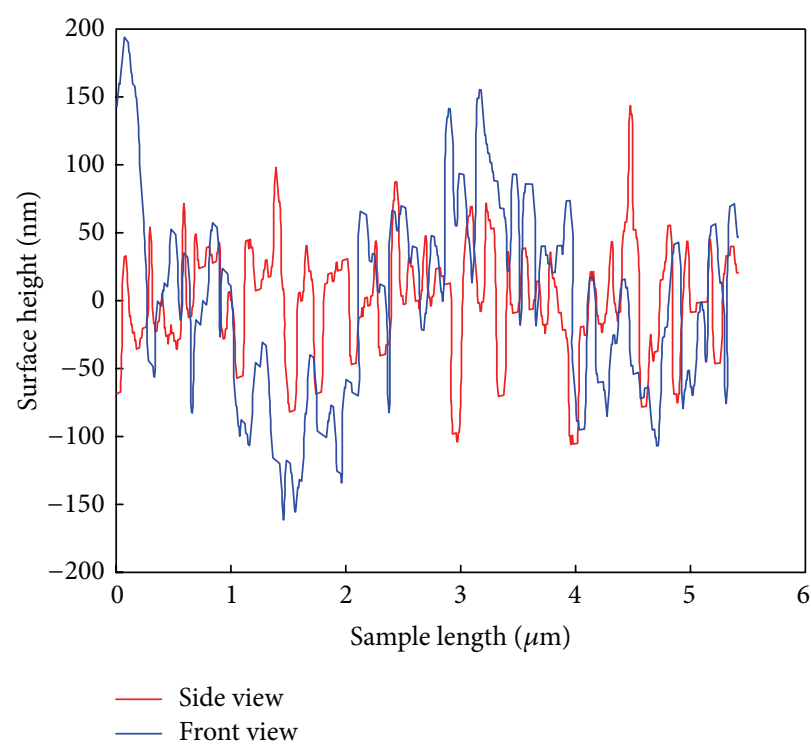

(a)

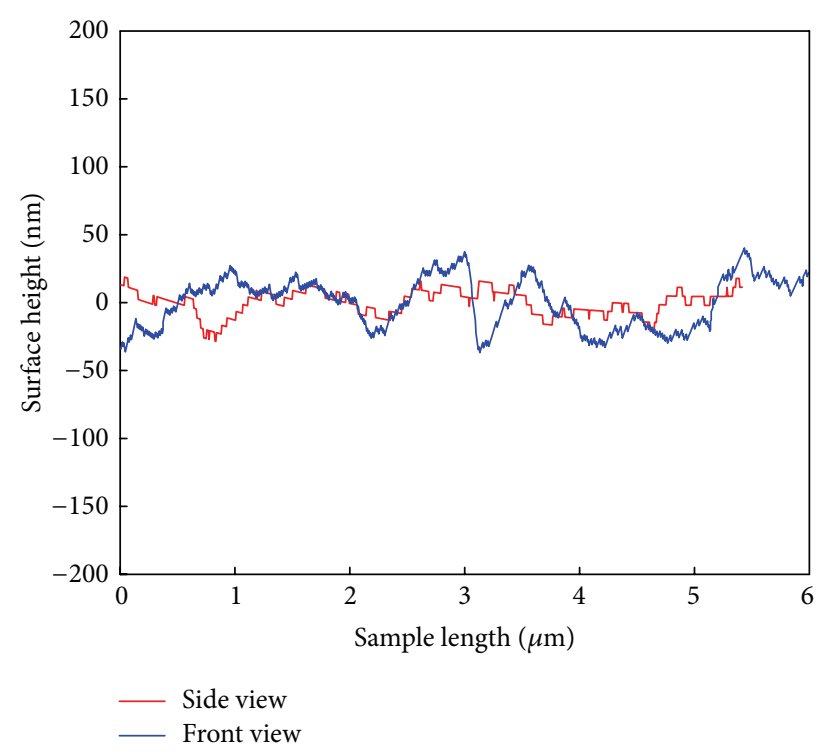

(b)

FIGURE 6: The profiles of the cylindrically hierarchical structures at front and side view produced at $\mathrm{Zn}^{2+}$ concentrations (a) $25 \mathrm{mM}$ and (b) $100 \mathrm{mM}$ of the growth solution.

optical fiber cores at cross section and further led to rougher profiles of the cylindrically hierarchical structures at front view.

The changes of both $\zeta_{\text {front }}$ and $\zeta_{\text {side }}$ values also demonstrate significant anisotropic surface morphologies of the cylindrically hierarchical structures. This is ascribed to the different curvature radii of the optical fiber cores at side and front view. As $\mathrm{Zn}^{2+}$ concentration of the growth solution increased, $\zeta_{\text {side }}$ values became larger steadily. This suggests that the profiles at side view turned from more random into rather correlated. It is ascribed to more uniformly aligned $\mathrm{ZnO}$ nanorods of larger diameters as well as less sizes of the pits among these $\mathrm{ZnO}$ nanorods. With $\mathrm{Zn}^{2+}$ concentration of the growth solution increasing, $\zeta_{\text {front }}$ values first decreased, then kept approximately stable, and eventually increased. As $\mathrm{Zn}^{2+}$ concentration of the growth solution was $25 \mathrm{mM}$, $\mathrm{ZnO}$ nanorods of smaller diameters radially were orientated from the surface of the optical fiber core. This resulted in larger sizes of the pits among the bundles of $\mathrm{ZnO}$ nanorods and further gave rise to larger values of $\zeta_{\text {front }}$. With $\mathrm{Zn}^{2+}$ concentration of the growth solution increasing continually, the diameters of $\mathrm{ZnO}$ nanorods increased whilst the sizes of the pits decreased, but the sum of both kept stable to some extent, so $\zeta_{\text {front }}$ values changed insignificantly. When $\mathrm{Zn}^{2+}$ concentration of the growth solution reached and was larger than $85 \mathrm{mM}, \mathrm{ZnO}$ nanostructures with even larger diameters appeared. This led to larger $\zeta_{\text {front }}$ values which approximated to $\zeta_{\text {side }}$ ones and isotropic surface morphologies of the cylindrically hierarchical structures emerged.

Most $\mathrm{Sk}_{\text {front }}$ values in Figure $7(\mathrm{~b})$ are less than $\mathrm{Sk}_{\text {side }}$ ones whilst the latter distributed symmetrically around zero (corresponding to a Gaussian rough profile). This suggests that the profiles at front view have more pits than those at side view whilst, on the profiles at side view, the number of pits is approximately equal to that of bumps. More radiation of $\mathrm{ZnO}$ nanorods on the optical fiber core at front view contributed much to this anisotropic behavior of the surface morphologies. Some $\mathrm{Ku}_{\text {front }}$ values in Figure 7 (b) were larger than 3 (corresponding to a Gaussian rough profile) whilst most $\mathrm{Ku}_{\text {side }}$ values were less than 3 . This means that the fluctuation ranges of the surface heights of the profiles at front view are larger than those at side view. These anisotropic surface morphologies of the cylindrically hierarchical structures are attributed to the radially orientated $\mathrm{ZnO}$ nanorods from the optical fiber core at front view. As $\mathrm{Zn}^{2+}$ concentration of the growth solution reached $100 \mathrm{mM}$, both $\mathrm{Ku}_{\text {front }}$ and $\mathrm{Ku}_{\text {side }}$ values are less than 3 and nearly equal to each other. This suggests that the surface morphologies of the cylindrically hierarchical structure at front and side view became isotropic. It is ascribed to the appearance of $\mathrm{ZnO}$ nanostructures instead of $\mathrm{ZnO}$ nanorods.

3.3.2. Characteristic Parameter PSD. Figure 8(a) demonstrates the PSD curves of both profiles of the cylindrically hierarchical structures at front and side view produced at $\mathrm{Zn}^{2+}$ concentration $25 \mathrm{mM}$ of the growth solution. Apparently, $\mathrm{PSD}_{\text {front }}$ curve in blue has two major spatial frequencies whilst $\mathrm{PSD}_{\text {side }}$ curve in red has one. Also, the $\mathrm{PSD}_{\text {front }}$ values corresponding to the major spatial frequencies are quite larger than related $\mathrm{PSD}_{\text {side }}$ ones. These suggest that the profile at front view is rougher than that at side view and the cylindrically hierarchical structure presented anisotropic surface morphology. Similar conclusion can also be drawn from the PSD curves of the profiles of the cylindrically hierarchical structure at front and side view formed at $\mathrm{Zn}^{2+}$ concentration $100 \mathrm{mM}$ of the growth solution in Figure 8(b). 


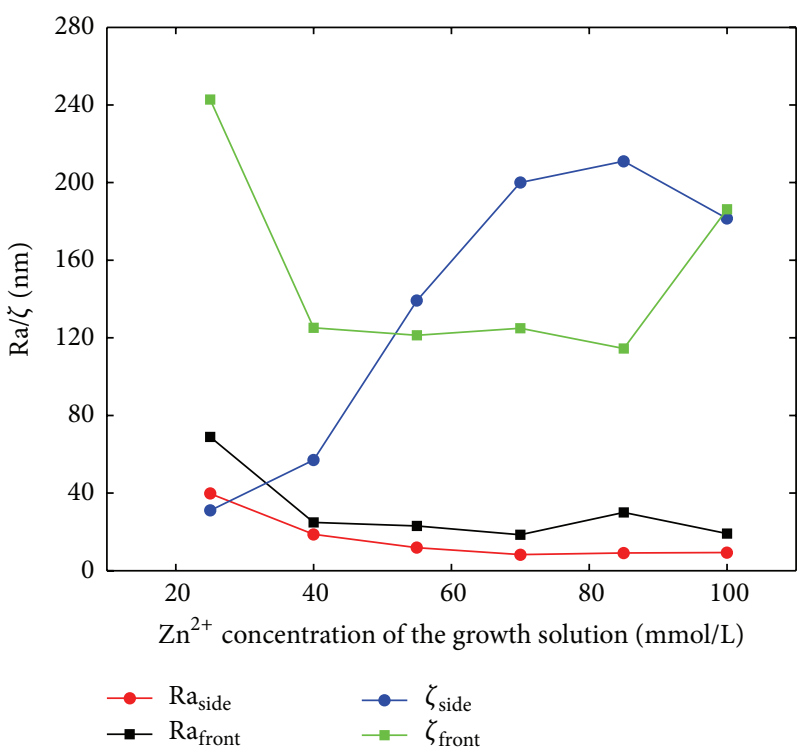

(a)

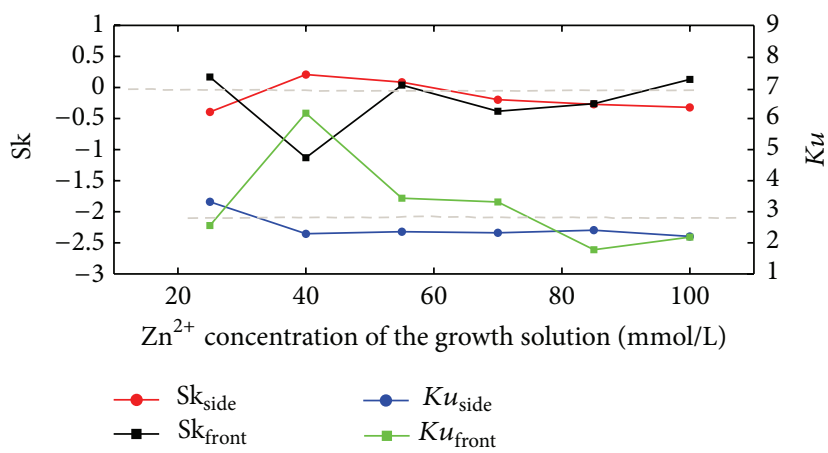

(b)

FIGURE 7: Effect of $\mathrm{Zn}^{2+}$ concentration of the growth solution on the characteristic parameters $\mathrm{Ra}, \zeta$ (a), and Sk, Ku (b) of the surface morphologies of the cylindrically hierarchical structures.

PSD curves in blue and in red in Figure 8(a) have longer tails than corresponding ones in Figure 8(b). This suggests that both profiles of the former cylindrically hierarchical structure at front and side view have wider spatial frequency ranges than those of the latter one. Furthermore, the PSD values corresponding to major spatial frequencies of the former cylindrically hierarchical structure are significantly larger than those of the latter one. These mean that the profiles of the former cylindrically hierarchical structure at front and side view are rougher than those of the latter one. The appearance of $\mathrm{ZnO}$ nanostructures instead of $\mathrm{ZnO}$ nanorods is attributed to this evolution of the surface morphologies of the cylindrically hierarchical structures.

\section{Conclusion}

With $\mathrm{ZnO}$ nanorods hydrothermally synthesized on manually assembled arrays of 2 and 3 layers of optical fiber cores with nominal pitches $250 \mu \mathrm{m}$ and $375 \mu \mathrm{m}$, the 3D arrays

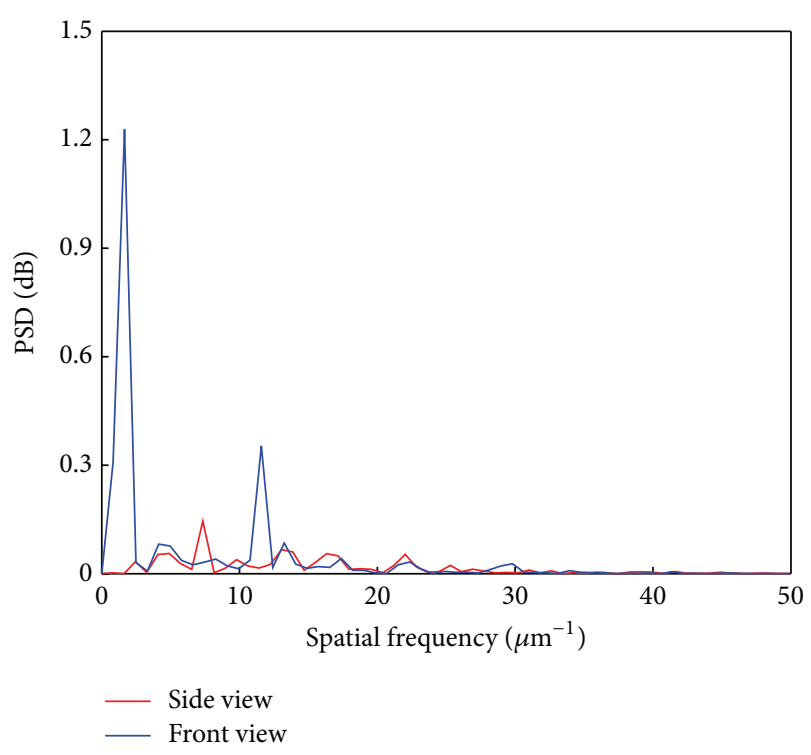

(a)

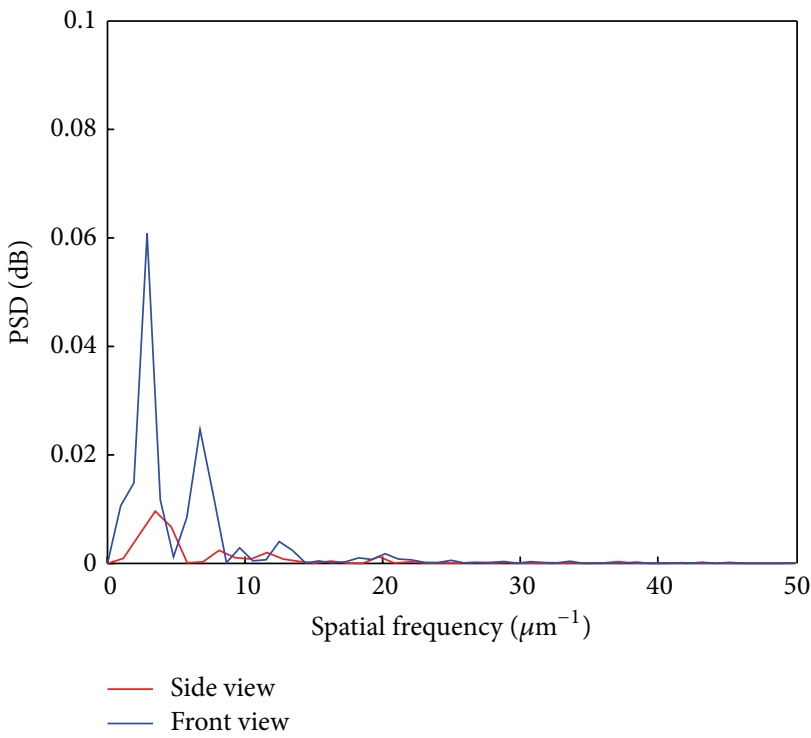

(b)

FIGURE 8: PSD curves of the profiles of the cylindrically hierarchical structures at front and side view produced at $\mathrm{Zn}^{2+}$ concentrations (a) $25 \mathrm{mM}$ and (b) $100 \mathrm{mM}$ of the growth solution.

of $\mathrm{ZnO}$ nanorod-based cylindrically hierarchical structures were constructed. More geometric parameters were introduced and the 3D arrays of cylindrically hierarchical structures were quantitatively characterized in detail. The characterization results suggest that the process capability of the manual assembling is sufficient and the quality of the $3 \mathrm{D}$ arrays of cylindrically hierarchical structures is acceptable. The anisotropic surface morphology of the cylindrically hierarchical structure was caused by the different curvature radii of the optical fiber core at front and side view and was affected significantly by $\mathrm{Zn}^{2+}$ concentration of the growth solution. The $3 \mathrm{D}$ arrays of $\mathrm{ZnO}$ nanorod-based cylindrically 
hierarchical structures have a potential to be used as enzymatic electrodes or photocatalytic reactors.

\section{Conflict of Interests}

The authors declare that there is no conflict of interests regarding the publication of this paper.

\section{Acknowledgments}

The authors would like to acknowledge the financial support from the NSFC Major Research Plan on Nanomanufacturing (nos. 51075324 and 91323303), the National Key Scientific Instrument and Equipment Development Projects of China (no. 2012YQ03026101), the National Key Basic Research Program of China (no. 2015CB057400), and the 111 Project (no. B12016).

\section{References}

[1] J. S. Jeong and J. Y. Lee, "Investigation of initial growth of $\mathrm{ZnO}$ nanowires and their growth mechanism," Nanotechnology, vol. 21, no. 47, Article ID 475603, 2010.

[2] P. Ji, T. Xu, M. He, and J. Li, "Simultaneous catalyst-free growth of highly oriented $\mathrm{ZnO}$ nanowires and microtubes," Journal of Nanoscience and Nanotechnology, vol. 13, no. 8, pp. 5919-5923, 2013.

[3] Z. L. Wang, "Zinc oxide nanostructures: growth, properties and applications," Journal of Physics Condensed Matter, vol. 16, no. 25, pp. R829-R858, 2004.

[4] Z. L. Wang and J. Song, "Piezoelectric nanogenerators based on zinc oxide nanowire arrays," Science, vol. 312, no. 5771, pp. 242246, 2006.

[5] H. Li, M. Zheng, S. Liu, L. Ma, C. Zhu, and Z. Xiong, "Reversible surface wettability transition between superhydrophobicity and superhydrophilicity on hierarchical micro/nanostructure $\mathrm{ZnO}$ mesh films," Surface and Coatings Technology, vol. 224, pp. 8892, 2013.

[6] T. T. Vu and G. Marbán, "Sacrificial template synthesis of high surface area metal oxides. Example: an excellent structured Fenton-like catalyst," Applied Catalysis B: Environmental, vol. 152-153, pp. 51-58, 2014.

[7] R. Yakimova, L. Selegard, V. Khranovskyy, R. Pearce, A. L. Spetz, and K. Uvdal, "ZnO materials and surface tailoring for biosensing," Frontiers in Bioscience, vol. 4, no. 1, pp. 254-278, 2012.

[8] T. T. Vu, L. del Río, T. Valdés-Solís, and G. Marbán, "Stainless steel wire mesh-supported $\mathrm{ZnO}$ for the catalytic photodegradation of methylene blue under ultraviolet irradiation," Journal of Hazardous Materials, vol. 246-247, pp. 126-134, 2013.

[9] J. Y. Kim, S.-Y. Jo, G.-J. Sun, A. Katoch, S.-W. Choi, and S. S. Kim, "Tailoring the surface area of $\mathrm{ZnO}$ nanorods for improved performance in glucose sensors," Sensors and Actuators B: Chemical, vol. 192, pp. 216-220, 2014.

[10] W.-X. Jing, L.-L. Niu, B. Wang et al., "Preparation and characterization of a $\mathrm{ZnO}$ nanowires-based cylindrical hierarchical structure," Chemical Journal of Chinese Universities, vol. 34, no. 7, pp. 1585-1590, 2013.

[11] F. Zhou, X. Li, J. Shu, and J. Wang, "Synthesis and visible light photo-electrochemical behaviors of $\mathrm{In}_{2} \mathrm{O}_{3}$-sensitized $\mathrm{ZnO}$ nanowire array film," Journal of Photochemistry and Photobiology A: Chemistry, vol. 219, no. 1, pp. 132-138, 2011.

[12] S. Kundu, "A facile route for the formation of shape-selective $\mathrm{ZnO}$ nanoarchitectures with superior photo-catalytic activity," Colloids and Surfaces A: Physicochemical and Engineering Aspects, vol. 446, pp. 199-212, 2014.

[13] H. Dai, Y. Zhou, Q. Liu et al., "Controllable growth of dendritic $\mathrm{ZnO}$ nanowire arrays on a stainless steel mesh towards the fabrication of large area, flexible dye-sensitized solar cells," Nanoscale, vol. 4, no. 17, pp. 5454-5460, 2012.

[14] S. Xu, C. Cheng, W. Guo et al., "Tuning the optical and electrical properties of hydrothermally grown $\mathrm{ZnO}$ nanowires by sealed post annealing treatment," Solid State Communications, vol. 160, pp. 41-46, 2013.

[15] B. Xiang, P. Wang, X. Zhang et al., "Rational synthesis of ptype zinc oxide nanowire arrays using simple chemical vapor deposition," Nano Letters, vol. 7, no. 2, pp. 323-328, 2007.

[16] I. Kazeminezhad and R. Yousefi, "Growth and characterization of $\mathrm{ZnO}$ (microdisks) $/ \mathrm{W}_{18} \mathrm{O}_{49}$ (nanorods) heterostructures," Solid State Sciences, vol. 14, no. 3, pp. 349-353, 2012.

[17] T. T. Vu, L. del Río, T. Valdés-Solís, and G. Marbán, “Tailoring the synthesis of stainless steel wire mesh-supported $\mathrm{ZnO}$," Materials Research Bulletin, vol. 47, no. 6, pp. 1577-1586, 2012.

[18] C.-J. Lin, S.-Y. Chen, and Y.-H. Liou, "Wire-shaped electrode of $\mathrm{CdSe}$-sensitized $\mathrm{ZnO}$ nanowire arrays for photoelectrochemical hydrogen generation," Electrochemistry Communications, vol. 12, no. 11, pp. 1513-1516, 2010.

[19] W. Jing, L. Niu, B. Wang, L. Chen, and Z. Jiang, "Simulation and $\mathrm{ZnO}$ nanowires-based generation of a hierarchical structure on an optical fiber core," Applied Surface Science, vol. 280, pp. 186192, 2013

[20] S. M. Usman Ali, O. Nur, M. Willander, and B. Danielsson, "A fast and sensitive potentiometric glucose microsensor based on glucose oxidase coated $\mathrm{ZnO}$ nanowires grown on a thin silver wire," Sensors and Actuators, B: Chemical, vol. 145, no. 2, pp. 869-874, 2010.

[21] L. Zhang and W. Jiao, "Preparation and performance of $\mathrm{ZnO}$ nanowires modified carbon fibers reinforced $\mathrm{NiFe}_{2} \mathrm{O}_{4}$ ceramic matrix composite," Journal of Alloys and Compounds, vol. 581, pp. 11-15, 2013.

[22] G. J. Ehlert and H. A. Sodano, "Zinc oxide nanowire interphase for enhanced interfacial strength in lightweight polymer fiber composites," ACS Applied Materials \& Interfaces, vol. 1, no. 8, pp. 1827-1833, 2009.

[23] M. H. Huang, S. Mao, H. Feick et al., "Room-temperature ultraviolet nanowire nanolasers," Science, vol. 292, no. 5523, pp. 1897-1899, 2001. 

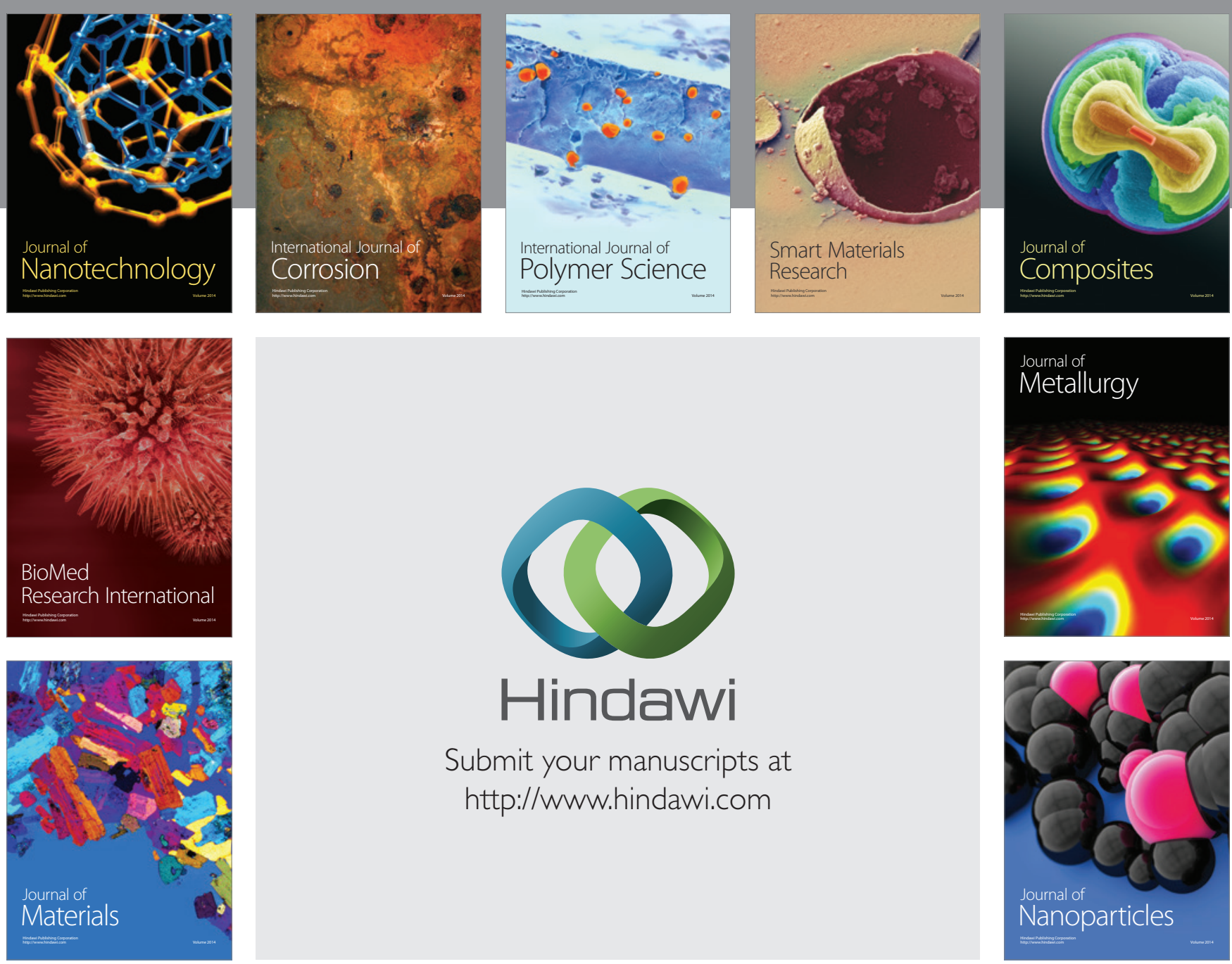

Submit your manuscripts at http://www.hindawi.com
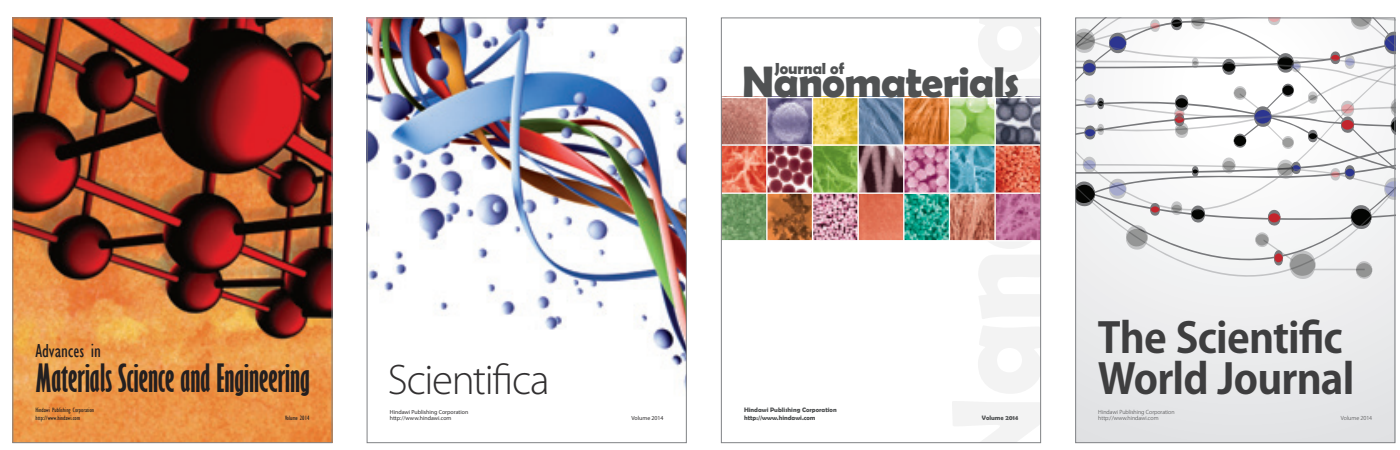

\section{The Scientific World Journal}
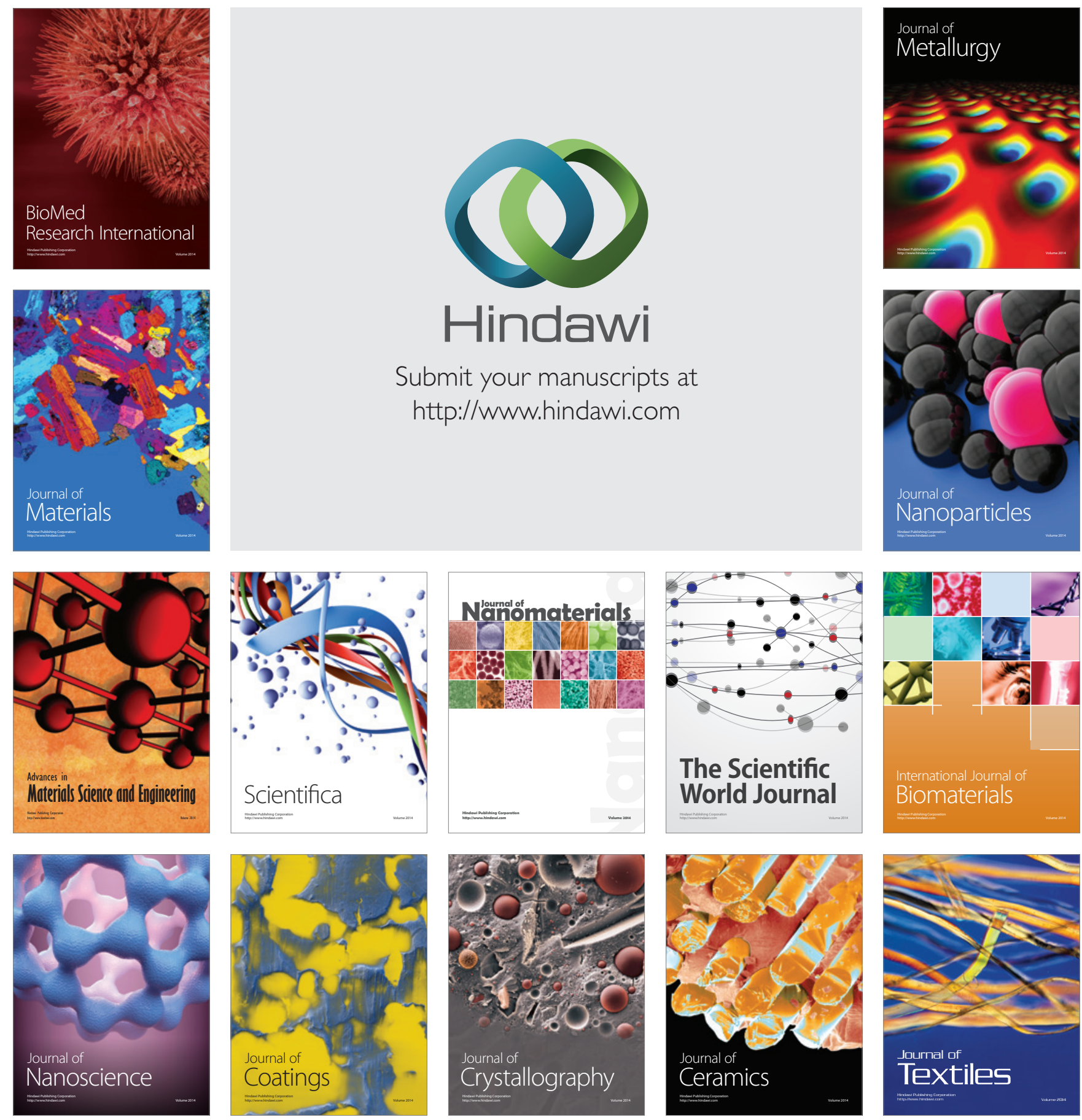